\title{
Robotic-Assisted Laparoscopic Ovarian Cystectomy during Pregnancy
}

\author{
Susanna Carter, M.D., ${ }^{1}$ Steven Depasquale, M.D., ${ }^{3}$ and Shawn Stallings, M.D. ${ }^{2}$
}

\section{ABSTRACT}

The use of robotic-assisted laparoscopic surgery (RALS) in gynecologic oncology is rising rapidly; however, the role of this modality in obstetrics has not been widely investigated. During pregnancy, the surgical management of adnexal masses is traditionally approached via laparotomy or laparoscopy. RALS offers a minimally invasive approach secondary to improved instrument dexterity and precision, 14-fold magnification, and 3-D imaging. For the pregnant patient, this translates into minimal manipulation of the gravid uterus, quicker recovery times, and potentially decreased maternal and fetal morbidity. Here we report six cases in which the da Vinci robotic surgical system (Intuitive Surgical Incorporated, Sunnyvale, CA) was used to perform an ovarian cystectomy during pregnancy. Pathology in all cases returned benign and each patient continued pregnancy without complications of surgery. In centers with the resources and adequately trained physicians, RALS offers the obstetric patient a safe and less invasive alternative to laparotomy or conventional laparoscopy. Although the advantages of robotic surgery are many, the limitations of this modality remain elevated equipment costs as well as the time investment necessary to train physicians.

KEYWORDS: Robotic surgery, adnexal masses, complications of pregnancy

Since gaining U.S. Food and Drug Administration approval for its use in gynecologic surgery in 1995, the da Vinci robotic surgical system (Intuitive Surgical Incorporated, Sunnyvale, CA) has quickly become an invaluable tool for the gynecologic oncologist. Likewise, as more general gynecologists become trained in robotic surgery, the role of this modality in pelvic surgery is expanding. There is a paucity of information in the current literature, however, on the use of robotic surgery in obstetrics. A review of the literature reveals one case report of a successful partial nephrectomy performed via robotic-assisted laparoscopic surgery (RALS) in a pregnant patient, ${ }^{1}$ and at our institution, there are two published cases of robotically placed transabdominal cervical cerclage in women currently pregnant and with a history of cervical incompetence. ${ }^{2}$ Here we report the first case series of six women who underwent successful robotic-assisted laparoscopic removal of an ovarian mass during pregnancy.

The incidence of ovarian masses in pregnancy is reported to fall between $1 / 81$ and $1 / 8000{ }^{3}$ Persistent adnexal masses in pregnancy can present a clinical dilemma secondary to the risks associated with both watching and removing the masses. Some studies have shown an incidence of cancer in persistent adnexal masses in pregnancy to range from 2 to $6 \%,{ }^{4}$ and therefore the general recommendation has been to remove those masses that persist at $6 \mathrm{~cm}$ or larger. ${ }^{5}$ Typically,
${ }^{1}$ Department of Obstetrics and Gynecology, ${ }^{2}$ Division of Maternal Fetal Medicine, University of Tennessee College of MedicineChattanooga; ${ }^{3}$ Chattanooga's Program in Women's Oncology, Chattanooga, Tennessee.

Address for correspondence and reprint requests: Susanna Carter, M.D., PGY-4, Department of Obstetrics and Gynecology, University of Tennessee College of Medicine-Chattanooga, 979 East Third Street, Suite 720, Chattanooga, TN 37403 (e-mail: sula7@earthlink.net).
Am J Perinatol Rep 2011;1:21-24. Copyright (C) 2011 by Thieme Medical Publishers, Inc., 333 Seventh Avenue, New York, NY 10001, USA. Tel: +1(212) 584-4662.

Received: November 4, 2010. Accepted: November 4, 2010. Published online: January 24, 2011.

DOI: http://dx.doi.org/10.1055/s-0030-1271220.

ISSN 2157-6998. 
the removal of such masses is achieved through either laparotomy or traditional laparoscopy. Despite early studies suggesting that operating in the second trimester reduced the incidence of first-trimester abortion or third-trimester preterm labor, the Society of American Gastrointestinal Endoscopic Surgeons now promotes the safety of laparoscopy in any trimester. ${ }^{6}$

At our institution, a collaborative effort between maternal-fetal medicine and gynecologic oncology has led to the introduction of a newer modality, the robotic surgical system, as a means of removing symptomatic pelvic masses in pregnancy. In an effort to potentially decrease maternal blood loss and reduce the incidence of conversion to laparotomy, the da Vinci surgical system was used to remove large persistent ovarian cysts from six obstetric patients. Each woman was referred to Chattanooga's Program for Women's Oncology (CPWO) secondary to a symptomatic or enlarging adnexal mass. In all six cases, the same gynecologic oncologist performed the operation. Each patient was counseled on the risks of removal including potential loss of the pregnancy as well as the risks of conservative management including rupture, torsion, preterm labor, and potential malignancy. Fetal heart tones were confirmed prior to and following surgery in each case.

All six RALS procedures were performed under general anesthesia. In each case, an open technique was employed for placement of the first laparoscopic trocar 6 to $10 \mathrm{~cm}$ above the umbilicus. This $10-\mathrm{mm}$ port was used for the 0 -degree endoscope. The abdomen was insufflated to an intra-abdominal pressure of $12 \mathrm{~mm}$ $\mathrm{Hg}$. Three to four additional port sites were chosen, and trocars were placed under direct laparoscopic visualization. These sites included two 8-mm ports for the robotic arms and one or two $10-\mathrm{mm}$ accessory ports. The robotic surgical system was then docked to the patient.

\section{CASE SERIES}

\section{Case 1}

A 46-year-old gravida 3, para 2 at 19 weeks' gestation was referred to CPWO in regards to an enlarging left adnexal mass. Discovered early in pregnancy, the $14-\mathrm{cm}$ mass had increased by $2 \mathrm{~cm}$ and was beginning to cause abdominal pain and shortness of breath. On exam, the mass was palpable in the upper abdomen and the uterine fundus was palpable at the umbilicus. After being counseled on the risks of conservative management including torsion and rupture as well as the possibility of malignant pathology, the patient elected to have surgery. At 20 weeks' gestation, she underwent a successful roboticassisted laparoscopic left ovarian cystectomy. Despite the advanced gestation and the size of the mass, the latter was removed with zero manipulation of the uterus.
Intraoperatively, the cyst was drained prior to removal with minimal spill. Pathology returned consistent with a benign mucinous cystadenoma. Estimated blood loss for the procedure was reported as $15 \mathrm{~mL}$. Operative time was 1 hour, 35 minutes. The patient was observed overnight, and discharged the following morning. Two weeks later at a follow-up appointment, the patient reported resolution of her pain and denied complications of her surgery.

\section{Case 2}

A 38-year-old gravida 3, para 2 was referred to CPWO at 14 weeks' gestation in consultation regarding a complex left ovarian cyst. The cyst was first discovered in the first trimester at her initial ultrasound and over the course of 6 weeks had grown $3 \mathrm{~cm}$. At the time of consultation, the mass measured $10 \times 6 \times 13 \mathrm{~cm}$ and was causing significant abdominal and pelvic pain. After discussion of the differential diagnosis ranging from benign cyst to ovarian malignancy as well as discussing options of removal versus close follow-up, the patient elected to have surgery. At 15 weeks' gestation, she underwent a robotic-assisted laparoscopic ovarian cystectomy. Intraoperative consultation with pathology revealed a benign mixed (mucinous and serous) epithelial cystadenoma. Estimated blood loss for the procedure was $10 \mathrm{~mL}$. Operative time for the case was 1 hour, 35 minutes. The patient was discharged home after an uneventful overnight hospital stay. At 17 weeks, the patient was seen for follow-up and complained of minimal discomfort at one of the trocar sights. She had a normal ultrasound at her primary provider that week, which revealed reassuring fetal growth and absence of the mass.

\section{Case 3}

A 19 -year-old gravida 1 at 13 weeks' gestation was referred to CPWO regarding her diagnosis of a large pelvic mass. Transvaginal ultrasound during the first trimester revealed a $15-\mathrm{cm}$ right ovarian mass. The patient denied symptoms yet on pelvic exam had a large, tender mass palpated in the posterior culde-sac. Given the size of the mass and its potential for encroaching on the rectum and enlarging uterus, the recommendation was made to remove it surgically. At 15 weeks' gestation, she underwent a successful roboticassisted laparoscopic right ovarian cystectomy. Intraoperatively, the cyst was ruptured to decompress it for removal. Operating room findings were consistent with a mature cystic teratoma, and the diagnosis was confirmed by pathology. Estimated blood loss for the procedure was $15 \mathrm{~mL}$. Operative time was 1 hour, 32 minutes. The patient recovered quickly and continued her pregnancy without complication. 


\section{Case 4}

A 25-year-old gravida 1 at 15 weeks' gestation presented to CPWO in consultation regarding a persistent ovarian cyst. Early in the pregnancy, she had undergone ultrasound-guided drainage of a right ovarian cyst. The pathology at that time revealed a benign serous cystadenoma. During the second trimester, the cyst fluid reaccumulated and was now causing significant abdominal pain. On exam, the mass was palpable above the umbilicus. The intention to remove the cyst robotically was explained; however, she was counseled on the possibility of a laparotomy. It was felt that the size and location of the mass could potentially impede trocar placement and visualization. Despite these potential obstacles, the patient underwent successful removal of a $16-\mathrm{cm}$ ovarian cyst via robotic-assisted laparoscopy. Estimated blood loss was recorded as "minimal," and pathology confirmed the original diagnosis of benign serous cystadenoma. Operative time was 1 hour, 26 minutes. The patient recovered quickly and reported no adverse symptoms at follow-up. One month later, an abdominal ultrasound confirmed appropriate fetal growth and absence of the mass.

\section{Case 5}

A 23-year-old gravida 2, para 1 at 18 weeks' gestation was referred to CPWO regarding a large ovarian mass. Measuring $18 \mathrm{~cm}$ in size, the cyst was causing considerable abdominal pain and the patient requested removal. The patient was counseled on the differential diagnosis ranging from benign cyst to ovarian carcinoma. The potential need for laparotomy was discussed secondary to the size of the mass. At 19 weeks' gestation, she underwent a successful robot-assisted laparoscopic ovarian cystectomy without complication. Estimated blood loss for the procedure was $15 \mathrm{~mL}$. Pathology returned consistent with a benign mucinous epithelial cystadenoma. Operative time for the case was 2 hours, 33 minutes. She continued her pregnancy to term without complication.

\section{Case 6}

A 21-year-old gravida 1 at 20 weeks' gestation presented to $\mathrm{CPWO}$ in consultation regarding a $15-\mathrm{cm}$ ovarian mass. Aside from mild nausea and vomiting, which the patient attributed to her pregnancy, she remained asymptomatic. After a discussion involving the differential diagnosis including benign and malignant pathology as well as the potential risk of torsion or rupture, the patient elected to have the mass removed. At 21 weeks' gestation, the mass was removed via robotic-assisted laparoscopy without complication. The pathology returned consistent with a benign mucinous cystadenoma. Estimated blood loss was minimal. Operative time was 3 hours, 16 minutes. At follow-up 2 weeks later, the patient denied complaints and reported a normal ultrasound confirming appropriate fetal growth. At 30 weeks' gestation, she had preterm premature rupture of membranes and was hospitalized until delivery.

\section{DISCUSSION}

These six cases have in common several aspects that serve to underscore the potential advantages of RALS in pregnancy. In each of these scenarios, the sheer size of these adnexal masses would have posed a significant challenge to a traditional laparoscopic approach. The robotic system allows 14 -fold magnification, a bioptic scope providing 3-dimensional imaging, and instruments with more than 500 degrees of motion. This translates into less if any manipulation of the gravid uterus, superior visualization, more precise dissection, and fewer conversions to laparotomy. Essentially, the robot provides laparoscopically the visualization and precision offered in an open case. Attempting to remove the larger masses through the traditional laparoscope could have potentially led to prolonged pneumoperitoneum, increased blood loss, or worse, conversion to laparotomy. Of note, these procedures were all performed at a lower level of intra-abdominal pressure than traditional laparoscopy would have allowed. Because of the improved dexterity of the instruments, ovarian conservation was achieved in all patients, making each case a true cystectomy. Additionally, though each ovarian mass in this series returned benign, the robotic approach provides the oncologist the tools necessary to complete a surgical staging at the time of cystectomy in the event a malignant process is identified. The six cases here demonstrate that robotic surgery is a safe and feasible alternative to laparotomy and conventional laparoscopy for the obstetric patient with a persistent, large, or symptomatic ovarian mass.

Although the advantages of RALS pertain primarily to the superior quality of surgical dexterity and visualization offered by the system, the cost of this modality has prohibited its mainstream acceptance. At 1.65 million dollars per system and an annual maintenance of 149,000 dollars, ${ }^{7}$ the robotic system is expensive and therefore available in limited centers. There is also a lengthy time investment necessary to adequately train surgeons, and this may lead to lost productivity in other areas. Another disadvantage of RALS, as with any newer modality, is the lack of data in the form of randomized controlled trials regarding its best implementation. Large studies are needed to truly compare cost-effectiveness, operating room times, blood loss, and outcomes between RALS, traditional laparoscopy, and laparotomy. Furthermore, despite its growing use in general gynecology, there are essentially no data on the use of this modality in 
obstetrics. Here we suggest that in centers where the technology and trained surgeons are available, RALS may provide a safe and feasible alternate approach to the obstetric patient with an ovarian mass.

\section{REFERENCES}

1. Park SY, Ham WS, Jung HJ, et al. Robot-assisted laparoscopic partial nephrectomy during pregnancy. J Robot Surg 2008;2:193-195

2. Wolfe L, DePasquale S, Adair CD, et al. Robotic-assisted laparoscopic placement of transabdominal cerclage during pregnancy. Am J Perinatol 2008;25:653-655
3. Whitecar MP, Turner S, Higby MK. Adnexal masses in pregnancy: a review of 130 cases undergoing surgical management. Am J Obstet Gynecol 1999;181:19-24

4. Sherard GB III, Hodson CA, Williams HJ, Semer DA, Hadi HA, Tait DL. Adnexal masses and pregnancy: a 12-year experience. Am J Obstet Gynecol 2003;189:358-362; discussion 362-363

5. Bozzo M, Buscaglia M, Ferrazzi E. The management of persistent adnexal masses in pregnancy. Am J Obstet Gynecol 1997;177:981-982

6. Society of American Gastrointestinal and Endoscopic Surgeons. SAGES Guideline for laparoscopic surgery during pregnancy. Surg Endosc 1998;12:189-190

7. Visco AG, Advincula AP. Robotic gynecologic surgery. Obstet Gynecol 2008;112:1369-1384 